\title{
Fortgeschrittenes NSCLC
}

Mit der zusätzlichen Gabe von Nintedanib zu Docetaxel konnte seit zehn Jahren zum ersten Mal eine Verlängerung des Gesamtüberlebens in der Zweitlinientherapie beim Adenokarzinom der Lunge ohne nachgewiesene therapierbare Mutation gezeigt werden. In der Placebo-kontrollierten Phase-III-Studie LUME-Lung 1 profitierten Patienten mit aggressivem Krankheitsverlauf besonders von dem Angiokinaseinhibitor.

Ein Großteil der Patienten mit Adenokarzinom der Lunge kann nicht von den zugelassenen, zielgerichteten Therapien profitieren, da entsprechende Mutationen nicht nachgewiesen werden. Die Therapieoptionen für diese Patienten sind begrenzt. In der Erstlinie wird in der Regel mit einer Platin-Doublette behandelt, in der Zweitlinie sind Docetaxel, Pemetrexed, Erlotinib sowie Nintedanib plus Docetaxel und neuerdings auch Ramucirumab plus Docetaxel zugelassen.

\section{Patienten mit aggressivem Tumorwachstum profitieren besonders}

In der LUME-Lung 1-Studie wurde gezeigt, dass die Kombination von Nintedanib (Vargatef ${ }^{\oplus}$ ) plus Docetaxel einer Docetaxel-Monotherapie überlegen ist. Der primäre Studienendpunkt,

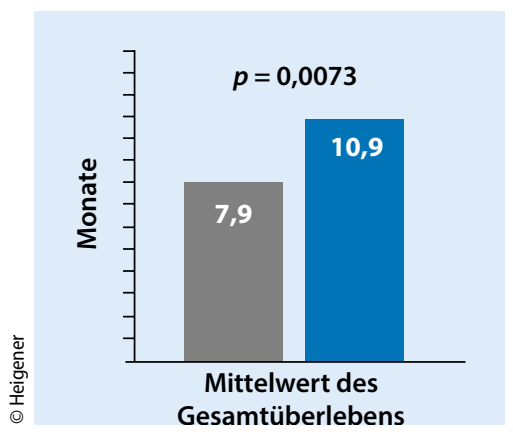

Abb. 1 ॥ 25-rozentige Risikoreduktion bei Patienten, die nach Erstlinientherapie progredient waren

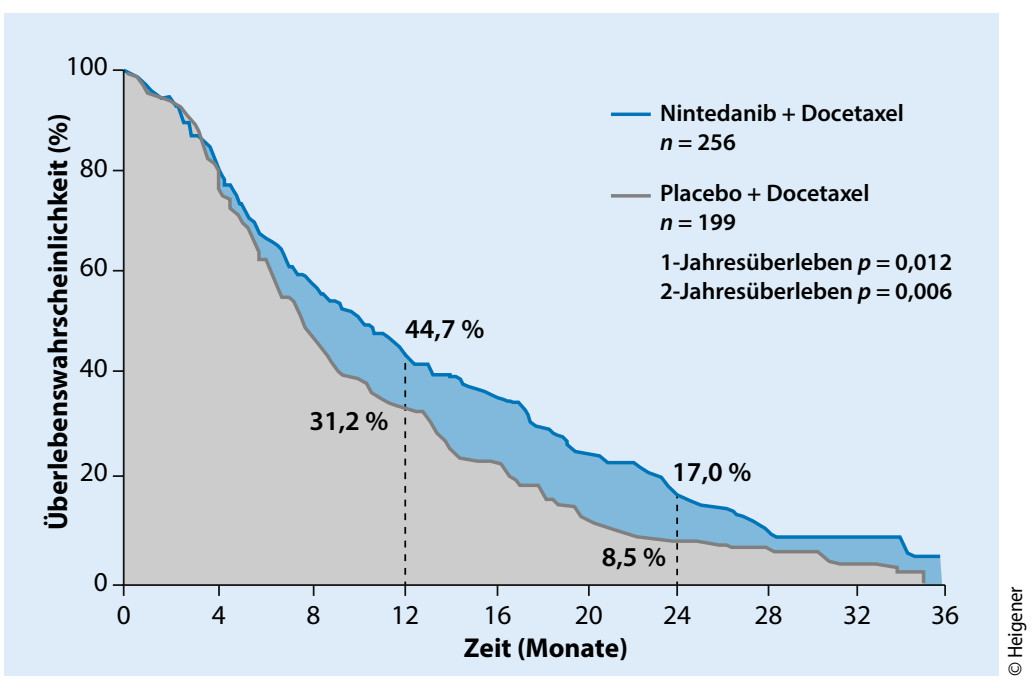

Abb. $2 \Delta$ Gesamtüberleben/Adenokarzinom-Patienten: Patienten mit Adenokarzinom und Progress innerhalb von 9 Monaten nach Beginn der Erstlinientherapie

eine Verlängerung des progressionsfreien Überlebens (PFS) wurde erreicht. Mit der zusätzlichen Nintedanib-Gabe wurde bei Adenokarzinom-Patienten erstmals eine signifikante Verlängerung des OS auf median mehr als ein Jahr (12,6 versus 10,3 Monate; $\mathrm{HR}=0,83$ ) erreicht, berichtete Dr. David Heigener, LungenClinic Grosshansdorf, in Bezug auf den sekundären Studienendpunkt, das Gesamtüberleben (OS): Die 1-Jahres-OS-Rate betrug 52,7 versus $44,7 \%$ und die 2-Jahres-OS-Rate 25,7 versus $19,1 \%$.

Auch bei Patienten mit aggressivem Krankheitsverlauf, die innerhalb von neun Monaten nach Beginn der Erstlinientherapie progredient waren, wurde eine 25-prozentige Risikoreduktion mit einem Median von 10,9 versus 7,9 Monaten beobachtet ( $\bullet$ Abb. 1, 2). Bei refraktären Patienten betrug die Risikoreduktion sogar $38 \%$. Je schlechter die Prognose, desto mehr profitierten also die Patienten, so Heigener.

Ine Schmale

Wien klin Mag 2016 · 19:63 DOI 10.1007/s00740-016-0102-9

Online publiziert: 5. April 2016

๑) Springer-Verlag Wien 2016
Quelle: Pressegespräch im Rahmen der Deutschen Krebskongress von Boehringer Ingelheim „Night of the experts: Zielgerichtete Therapien beim NSCLC", 24. Februar 2016 , Berlin 